\title{
BIOPHYSICS OF NERVOUS EMBRYO-FETAL DEVELOPMENT
}

\author{
Arturo Tozzi \\ Center for Nonlinear Science, Department of Physics, University of North Texas, Denton, Texas, USA \\ ASL Napoli 2 Nord, Distretto 45, Caivano, Naples, Italy \\ tozziarturo@libero.it \\ arturo.tozzi@alsnapoli2nord.it
}

The dynamical processes of living systems are characterized by the cooperative interaction of many units. This claim enables us to portray the embryo-fetal development of the central and peripheral nervous systems in terms of assemblies of building blocks. We describe how the structure and arrangement of nervous fibers is - at least partially - dictated by biophysical and topological constraints. The far-flung field of soft-matter polymers/nematic colloids sheds new light on the neurulation in mammalian embryos, suggesting an intriguing testable hypothesis: the development of the central and peripheral nervous systems might be correlated with the occurrence of local thermal changes in embryo-fetal tissues. Further, we show a correlation between the fullerene-like arrangement of the cortical microcolumns and the FrankKasper phases of artificial quasicrystals assemblies. The last, but not the least, we explain how and why the multisynaptic ascending nervous fibers connecting the peripheral receptors to the neocortical areas can be viewed as the real counterpart of mathematical tools such as knot theory and braid groups. Their group structure and generator operations point towards a novel approach to long-standing questions concerning human sensation and perception, leading to the suggestion that the very arrangement and intermingling of the peripheral nervous fibers contributes to the cortical brain activity. In touch with the old claims of D'Arcy Thompson, we conclude that the arrangement and the pattern make the function in a variety of biological instances, leading to countless testable hypotheses.

KEYWORDS: soft matter; liquid crystals; braid group; anyon; brain; microcolumn.

Development of multicellular living beings involves morphogenetic processes shaping embryo-fetal structures through self-organized activities of pluripotent stem cells (Veenvliet et al., 2020). The orchestrated movement of cellular groups involves genetic as well as mechanical and molecular interactions between cells and their environment (Thompson 1992; Barriga et al., 2018). Migrating cells respond to various stimuli, such as cortical tension, luminal pressure and size (Chan et al., 2019), local changes in tissue architecture (Box et al. 219), as well as topographical, adhesive and chemoattractant cues (Dai et al., 2020), duplication of existing regions (Kebschull et al., 2020), topological templates (Dabaghian et al., 2014). For example, changes in substrate stiffness triggers collective cell migration, suggesting that tissue mechanics combines with molecular effectors to coordinate morphogenesis (Barriga et al., 2018). Here we focus on the morphogenetic dynamical processes characterized by cooperative interaction and collective cell migration of numerous cellular units (Lovecchio et al., 2012). Setting aside the above-mentioned mechanisms of embryogenesis and cell migration, here we describe unnoticed biophysical/topological processes underlying the very structure of vertebrates' systems. In particular, we will focus on the embryo-fetal development of the central and peripheral nervous system, since the growth of the neuronal tissue is linked with the growth of a wide range of different structures.

Brain-derived signals are involved in the regulation of the whole embryogenesis, providing long-range cues among distinct structures. To provide an example, the multipotent neural crest cells are transient embryonic progenitors that describe stereotypical branching transcriptional trajectories and develop into different tissues ranging from craniofacial bones to glia, as well as peripheral neurons and autonomic and sensory nervous systems (Soldatov et al., 2019). During the embryonic development, unexpected interactions have been found between the nervous system and numerous craniofacial and trunk skeletal elements (Herrera-Rincon et al., 2017). Multipotent Schwann cell precursors detach from their nerve fibers commitment to become mesenchymal, chondroprogenitor and osteoprogenitor cells (Xie et al., 2019). Also, a link has been found between two apparently unrelated processes -gastrulation and neural crest migration- via changes in tissue mechanics (Barriga et al., 2018).

We introduce three novel feasible mechanisms to explain the embryo-fetal development of the nervous system. First, we portray the embryonal neurulation in terms of liquid/crystalline phase of condensed colloidal self matter. Second, we describe the development of cortical microcolumns in terms of fullerene-like self-aggregates. Third, we appraise the description of the intertwining peripheral nerves and the intricate grey/white matter in terms of knot theory and braid groups. Once illustrated these novel biophysical approaches to neuronal developmental issues, we show how they lead to a series of testable hypotheses. 


\section{SOFT MATTER POLYMERS AND EMBRYONAL NEURULATION}

The cellular structures can be viewed as building blocks characterized by liquid/crystalline phases of condensed soft matter where order and fluidity coexist (Qian et al., 2017). Mechanisms for intracellular organization within distinct compartments inside the cell -known as liquid-liquid phase separation- have been recently studied not just in eukaryotes, but also in bacteria (Ladouceur et al., 2020). Phase transitions drive proteins and other molecules to aggregate into cytoplasmatic and nuclear condensed fluid bodies, leading to nonuniform localization patterns and subcellular compartmentalization. These biomolecular condensates include membrane protein clusters, cytoplasmic P granules (Brangwynne et al., 2009), nucleolus, histone locus body, heterochromatin domains (Strom et al., 2017), protoplasmic gelation (Nguyen et al., 2020), amyloid-like assemblies (Boke et al., 2016), intrinsically disordered mixed-charge domains (Greig et al., 2020). Also, the self-replicating active liquid crystals of surface-attached bacterial biofilms display both local cellular order and global biofilm architecture generated by liquid crystals relationships (Hartmann et al., 2019). Liquid-phase condensates can be viewed as reaction centers where some components become enriched for processing or storage within cells. To provide an example, Garcia Quiroz et al. (2020) found that the keratinocytes of the stratified squamous epithelium undergo a vinegar-in-oil type of liquid-liquid phase separation, crowding the cytoplasm with increasingly viscous protein droplets that drive squame formation.

Compounds made of "liquid crystals" display properties between conventional liquids and solid crystals that can be experimentally studied in vitro. Mundoor et al. (2018) produced building blocks of a molecular-colloidal liquid crystal formed by micrometer-long inorganic silica-coated disks dispersed in a crystalline fluid host composed of molecular rods. Field-induced motion caused by magnetic fields elicited colloidal interactions between disks in nematic hosts and generated various symmetric conformations with different tangential surface orientations (Figure 1A) (Mundoor et al., 2018). Within a range of temperature and concentration, the freely diffusing rods ordered orthogonally to the solvent molecules, producing a biaxial liquid crystal. Mundoor et al. (2021) demonstrated that dispersion of isotropic charged colloidal disks in nematic host composed of molecular rods produces nematic, smectic and columnar organizations (Figure 1A). While regular polymeric materials respond in linear fashion to external stimuli such as high- temperature material softening, liquid crystals polymers display nonlinear and much faster macroscopic changes (Iqbal and Samiullah, 2013). Therefore, under the proper biophysical constraints, it is feasible to realize low-symmetry condensed matter phases in systems with building blocks of dissimilar shapes and sizes. That's what happens during the embryofetal differentiation of the central nervous system, where the bodily architecture recalls isotropic, nematic and smectic columnar arrangements (Chen et al., 2017). See Figure 1B for further details. It is noteworthy that biaxial nematics can be produced either through long inorganic nanorods and short organic molecules, or boardlike molecules, or component mixtures, paving the way to a next-to-come studies focused on organic structures. Experimental clues point towards a relationship between biological elements (in our case, the nervous elements) and phase changes. Recent data suggest that the assembly of the developing synaptic active zone requires the liquid phase of the scaffold molecules (McDonald, 2020).

In sum, we suggest to investigate the relationship between the dynamical processes leading to liquid crystals arrangements and the formation of the embryonic neural structures. To further scrutinize this hypothetical correlation, we suggest a testable hypothesis in the next paragraph.

A testable hypothesis. It is worth mentioning that the collective phenomena of colloidal interactions between disks in nematic hosts described by Mundoor et al. (2018 and 2019) are thermotropic, i.e., temperature-driven and the temperature-dependent. Increases in temperature in such thermally reconfigurable monoclinic nematic colloidal fluids lead to unusual transitions towards more-ordered states. Symmetry-breaking phase transitions occur when temperature is lowered, leading at first to transition from isotropic liquid to nematic phases and then to liquid-crystal smectic phase (Qian et al., 2017). In touch with the classical polymer physics, Kießling et al. (2013) observed a systematic deformation of the viscous cellular matter upon temperature changes. This enables us to draw an intriguing testable hypothesis that correlates differences in cellular temperature and developmental outcomes of the nervous system. Variations in thermal conductivity within biological cells point towards the occurrence of transient temperature spikes and heat flux for short-distance thermal signaling (Sotoma et al., 2021). A role for non-stationary local fluctuations of cellular temperature merits exploring. The neocortex displays thermal gradients observed at many spatiotemporal scales (Wang et al., 2014): in particular, Tanimoto et at. (2016) quantified the temperature difference in a human derived neuronal cell lines and concluded that temperatures in cell body and neurites are different. It is well-known that membrane patch temperature impacts neuronal activity through modulation of the opening and closing rates of ion channels (Xu et al., 2019). Local changes in thermal properties modulate presynaptic and postsynaptic events, sensory stimuli, memory encoding and behavioral changes (Long and Fee, 2008; Kalmbach and Waters, 2012). Thermodynamic arguments point towards a relationship between changes in temperature and message content, since modifications in thermic flows are correlated with variations in thermodynamic/information entropies (Jizba and Arimitsu, 2001; Jizba, 2015). Fluctuations of thermic properties across biological regions can be absorbed into the thermodynamic frameworks of dynamical systems theory (Tozzi and Peters, 2017). This means that the critical slowing related to decreases in temperatures of living systems is mandated by any system which provides an effort to minimize its own energetic expenditure. 
Therefore, the development of the central and peripheral nervous system could be linked with the occurrence of local thermal changes in embryo-fetal tissues. Therefore, changes in local temperature in murine embryonic models might lead to anatomical modifications in the final shape of their central and peripheral systems.

A

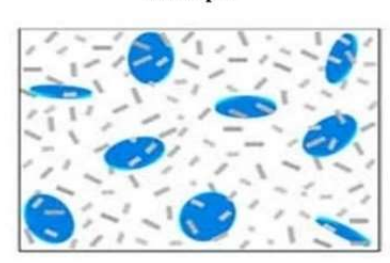

B

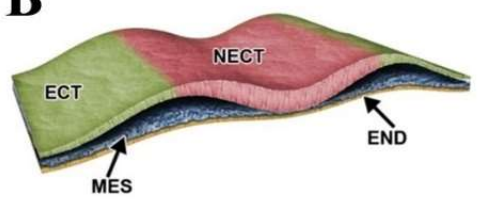

Nematic

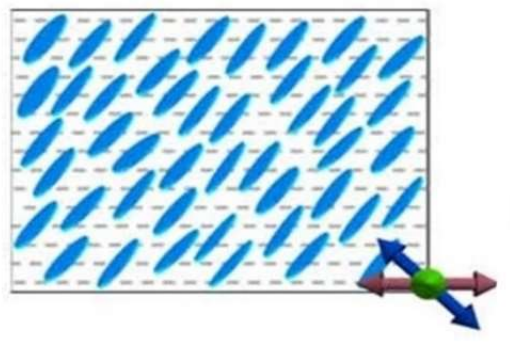

E8.0

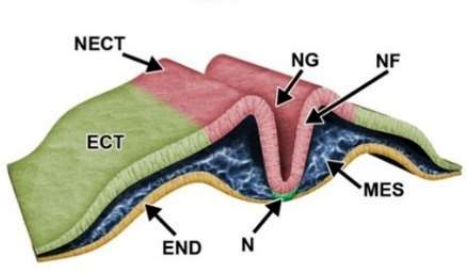

Smectic
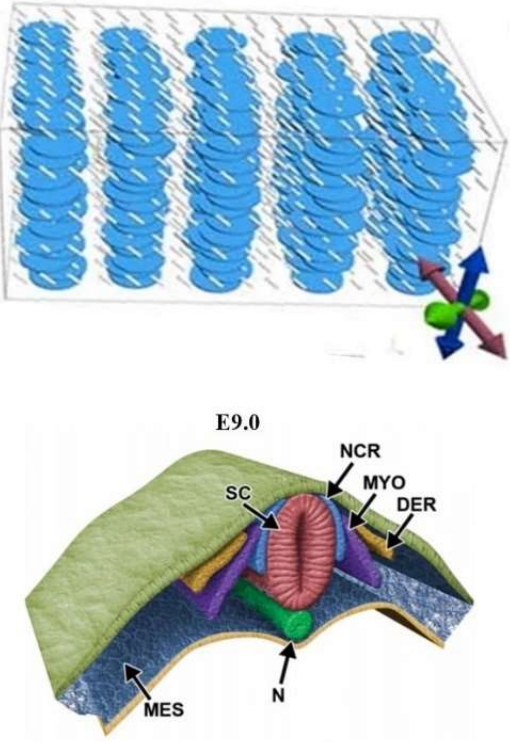

Figure 1. Comparison of two seemingly distant phenomena: the liquid crystal phases and the embryonic development of the nervous system. Figure 1A. Mixtures of molecular/colloidal rods and disks of fluid condensed matter give rise to the formation of temperature-dependent columnar chains displaying different uniaxial symmetries. Depending on the structural arrangement, we achieve isotropic, nematic and smectic liquid crystals. Modified from Mundoor et al. (2021). Figure 1B. Schematic transverse sections of neurulation in the mouse embryo at different early stages of development. While the primitive confined neuroectoderm at E.75 recalls isotropic liquid crystals, the converging neural folds at E8.0 remind the arrangement of nematic liquid crystals. In turn, the spinal cord at E.9.0 evokes the typical arrangement of smectic liquid crystals. Legenda: E: embryonic age in day; DER: dermatome, ECT: ectoderm, END: endoderm, MES: mesoderm, MYO: myotome, N: notochord, NECT: neuroectoderm, NF: neural folds, SC: spinal cord. Modified from Chen et al. (2017). 


\section{FULLERENIC-LIKE SELF-ASSEMBLIES AND MATURE CORTICAL MICROCOLUMNS}

In dynamical systems, simple elements spontaneously aggregate into larger, ordered structures, giving rise to different self-sustaining waves such as traveling, rotating, standing and reflected waves/spirals (Bhattacharya and Iglesias, 2019). In non-equilibrium systems characterized by self-organization of collective active particles, input-driven local fluctuations lead to the emergence of larger-scale ordering (Chvykov et al., 2021). This is the case not just of artificial devices such as shape-changing robotic active matter, but also of biological assemblies (Linneweber et al., 2020). For example, cellular self-organization promotes follicle pattern in the avian skin (Shyer et al., 2017), while cooperative sets of firing neurons exhibit temporal complexity and emergence of criticality (Lovecchio et al., 2012). Self-organization generates many processes, such as the above-mentioned gathering of colloids in liquid crystals, supramolecular assemblies of metal alloys, gold nanocrystal superlattices, proteins, viruses, nucleic acids, nervus structures (Tozzi 2015; Montis et al., 2021). In particular, crystallization may produce unusual self-assemblies of small and rigid organic molecules. In case of hyrochloride salts of fampridine, the crystallographic aggregates consist of Frank-Kasper (FK) phases, i.e., a quasicrystal combination of periodic structures and aperiodic tiling lacking translational symmetry (Montis et al., 2021). Inside a dense liquid phase, supramolecular assemblies can spontaneously aggregate to form FK structures of either spherical aggregates, or polyhedra with various coordination numbers (Montis et al. 2021).

Artificial materials subjected to Frank-Kasper (FK) phases such as the hyrochloride salt of fampridine can generate fullerenic-like self-assemblies (Montis et al. 2021). The same fullerenic structures have been recently proposed to explain some features of the cortical microanatomy. Indeed, Tozzi et al. (2017) showed that the tubular cortical microcolumn, i.e., the embryological, anatomical and functional basic unit of the brain, can be flattened to form fullerene-like two-dimensional lattices equipped with about 80 nodes corresponding to pyramidal neurons. Therefore, both artificial and natural self-assembled quasicrystal structures share a fullerene-like lattice grounded on simple topological changes.

In sum, the spontaneous occurrence of peculiar self-assembled artificial structures suggests a comparison with the design of natural organic crystalline materials such as the nervous structures.

\section{BRAID GROUPS, NERVE FIBERS AND SOMATOTOPIC MAPS}

In the previous paragraphs, we discussed how soft crystals interactions and self-assemblies could be involved in the development of embryo-fetal structures. Here the focus is tuned on the anatomical intertwining of nervous fibers in both central and peripheral nervous systems. We introduce new players: knot theory and braids. Knot theory provides topological tools for cataloguing different knots. Knots can be transformed one into another via a deformation of threedimensional space upon itself and summed in assemblages equipped with commutative and associative properties (Cromwell, 2004). A braid is a collection of strands between two parallel planes (Artin 1947). Braids are termed isotopic when, keeping their endpoints fixed, can be twisted into each other without cutting the strands. The operations of composition allow braids to be joined to achieve new ones. Given a set of braids with a fixed number of strands, its group structure is provided by generators and fusion rules such as, e.g., associativity, crossing, braiding/unbraiding, intertwining, composition and sequence of elementary braids, and so on. In algebraic topology, a well-established link does exist between braids and knots. Is it always possible to transform a knot into a closed braid: for example, Alexander theorem states that every knot or link in three-dimensional Euclidean space is the closure of a braid (Alexander 1923). Nevertheless, the correspondence between knots and braids is not one-to-one, because a single knot may have many braid representations. The Markov theorem provides the moves to relate closed braids representing the same knot type (Birman 1974).

Biology gives several examples of living structures that might be counterparts of mathematical knots and braids. Tissue morphogenesis is produced by coordinated regional changes in cell shape driven by localized contractions of actomyosin "braids" (Bailles et al., 2019). Frieden and Gatenby (2019) demonstrated wire-like flow of electrons and ions along cytoskeletal elements conveying messages from the cell membrane to the nucleus. The three-dimensional space where neural connexions are formed is severely constrained by physical factors such as the anatomical overlap of neuronal arbors and the available axonal space to make synapses. Reimann et al. (2017) found that the average overlap between the dendritic and axonal arbors of different types of neurons determines neuron-type specific patterns of distance-dependent connectivity, since higher-order connexions depend on the diverse branching patterns of individual arbors of the same neuronal type.

Braids, knots and the nervous system. Incorporating the mathematical/topological perspective of knot theory in neuroscience appears particularly relevant for understanding brain functions such as, e.g., how the brain represents and processes the environmental stimuli. In our framework, the arrangement of nervous fibers in an example of isotopic braids which describes the evolution of a multi-particle system equipped with two spatiotemporal ends: a beginning and an end. Just like the knots are embeddings of closed lines in the three-dimensional space and cannot be reduced to simple circles by a continuous deformation ( $\mathrm{Wu}, 1992)$, the nerves are structures that do not disentangle completely 
after being pulled from both ends. It is easy to show that the nervous fibers connecting distinct structures of the peripheral and the central nervous systems are shaped as braids. To provide an example, consider the sensory inputs from the external world detected by the peripheral nervous fibers: they follow a multisynaptic, ascending path towards the higher areas of the central nervous system. See Figures 2A-B for further details. This means that, if we term "braid group" the whole nervous tract between peripheral receptors and cortical areas, we are allowed to term "knots" the intermediate anatomical/functional multi-synaptic steps. Figure 2C tells us that also the anatomical nervous structures studied by tractography can be described in terms of braids. Our suggestion is twofold: a) simple changes in location and arrangement of nerves could explain the activity of the central nervous system; b) the external inputs follow specific nervous paths which can be described in the mathematical terms of braid groups.

What for? In the last paragraph, we concluded that the intricate paths detectable in the nervous system can be viewed as braids and knots. The next step is to answer to the crucial question: what for? We are fully aware that the overwhelming complexity of the mammalian nervous system makes it extremely difficult to map nervous anatomical structures to mathematical manifolds equipped with knots and braids. Given the objective obstacle to find the proper group generators, why should we care to describe the nervous paths in the tricky terms of braid groups? What are the (methodological, philosophical, explanatory, medical) benefits? In the sequel we describe the multiple advantages of this approach.

The existence of a theoretical link between the anatomical conformation of the nerves (i.e., knots and braids) and the brain activity would mean that different braiding rules might give rise to different nervous functions. Braid groups could be the key to explain the differences among different sensitive cues, since our framework suggests that various braids conformations lead to distinct computational processes/properties of every sensory cortex. Fully different perceptions such as the olfactive, auditory, tactile, visive perceptions could be explained by the distinct configurations of the braids that link the external receptors to the corresponding sensitive cortex. It is well-established that neural pathways are topographically organized, giving rise to maps that are preserved from the periphery to the cortex (Imam and Finlay, 2020). This could be explained by the occurrence of isotopic braids in nervous fibers: since the strands between the starting and the ending points (i.e., the nervous fibers between the peripheral receptor and the corresponding cortical projection) display the same intermingled conformation, they must carry the same message. Our hypothesis does not strictly require that the external message stands in 1:1 relationship with the corresponding cortical area: due to the braid rules, the relationship between the two extremities of the braids can be either injective, or surjective, bijective, and so on. Our hypothesis suggests that the nervous arrangement of the cortical somatotopic maps can be correlated with the arrangement of the peripheral nervous fibers. This means that - at least a part of- the human brain diseases might depend on the anatomical configuration of peripheral nerve fibers. Instead of looking for alterations in the central nervous systems, it would be reasonable to look for neuropathological features in the braids and knots of the peripheral nervous fibers. 

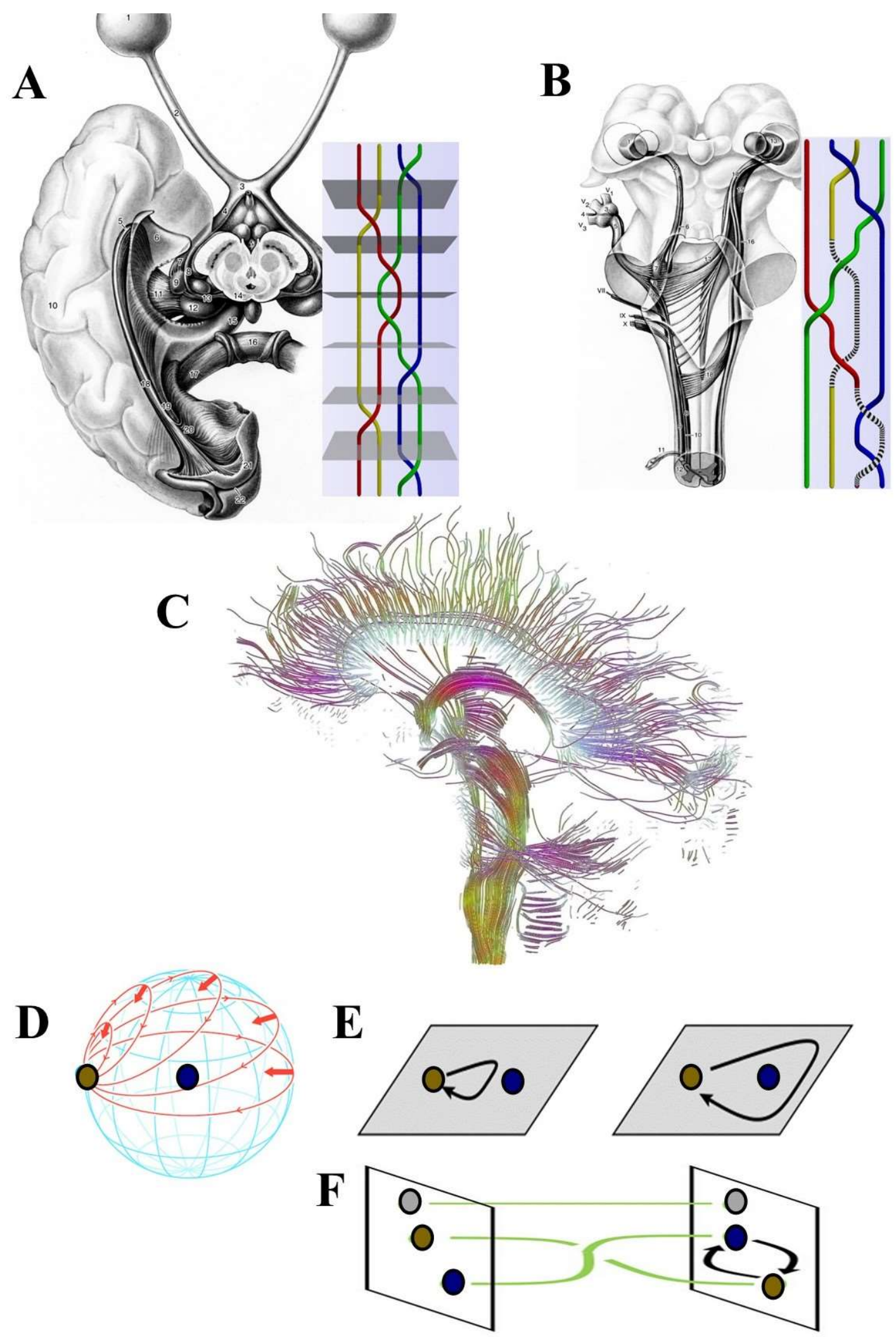

Figure 2A-B. Comparison of nervous connexions in the central/peripheral nervous systems and mathematical examples of braid groups. The Figures illustrate the retino-geniculo-cortical projection in a ventral view (Figure A), the central connections of the trigeminal nerve in a sagittal view (Figure B) and their hypothetical braid counterparts. Modified from: http://matematita.science.unitn.it/braids/summary.html and Nieuwenhuys et al. (2007). Figure C. Also 
the anatomical nervous structures detectable by tractography can be described in terms of braids. Modified from: http://www.sci.utah.edu/ gk/DTI-data/

Figure D-F. Different swaps between particles moving in different dimensions. Figure D. Feasible paths in three dimensions. When a particle loops around another inside a three-dimensional manifold, the loops are highly constrained, because they topologically shrink to a single point. Figure E. Feasible paths in two dimensions. Two topologically distinct paths may occur inside a two-dimensional manifold. On the left, the particle's path shrinks to a single point like the three-dimensional case. On the right, a particle loops around another, so that the loop is get caught by the other particle. This means that the loop on the right cannot shrink to a single point: a non-trivial path occurs here, topologically distinct and less constrained than the path depicted in the left figure. Figure F. Two-dimensional paths achievable through braid groups' operations. The events of loops get caught by other particles can be described in terms of braid groups, since the particles' paths form braids in $2+1$ dimensions.

\section{CONCLUSIONS}

We investigated the biophysical mechanisms that shape the structure and the activity of biological entities, suggesting that straightforward changes in the arrangement of nerves location might elucidate the activity of the central nervous system. We described how microscopic and macroscopic embryo-fetal and adult nerve fibers can be assessed in terms of braids. The latter are made of supramolecular assemblies generated by random walks taking place inside the viscous fluid of the nervous milieu. The connexions and the entanglement of their extremities give rise to reversible or irreversible topological knots, respectively standing for labile functional activities and stable anatomical tracts.

Taking a much more speculative approach, we may consider the cortical layer as a two-dimensional, instead of a threedimensional manifold. The brain activities involving different cortical areas could be described in terms of topologically distinct paths of particles traveling inside two-dimensional manifolds. Since the paths followed by two or more particles vary depending on the dimensions of the phase space where their movements take place, different swaps between particles are feasible in different dimensions. Figures 2D-F illustrate a few examples of particles' movements in various dimensions. Note that the swap between the blue and the red particles in Figure 2F may occur clockwise or counterclockwise: in case of a clockwise swap, we state that an undercrossing occurs; in case of a counterclockwise swap, we state that an overcrossing occurs. In the same vein, the mingling of peripheral nerve fibers in multisynaptic paths can be described as undercrossings and overcrossings. The last, but not the least, a recently described theoretical concept comes into play: field patterns. Field patterns are the paths followed by spacetime microstructures when a propagating disturbance concentrates on a distinctive line (Milton and Mattei, 2017). The various "braids" formed by field patterns with different spatial shifts do not interact, but rather evolve as if they live in separate dimensions. The addition of a tiny nonlinear term to wave equations gives rise to interactions between field patterns in their multidimensional spaces. This moves us into the realm of abelian and non-abelian anyons, i.e., intermediate particles between the Fermi Dirac statistics of fermions and the Bose Einstein statistics of bosons. But that's another story...

As a final remark, we would like to emphasize that our experimental suggestions are technically achievable with the current technology. The recent availability of artificial embryonic trunk-like structures from mouse stem cells' aggregates (Telley et al., 2019) provides the exciting possibility to study in vitro the complex developmental processes and to test our fully novel approaches to the mechanisms of nervous development.

\section{REFERENCES}

1) Alexander J. 1923. A lemma on a system of knotted curves. PNAS, 9 (3): 93-95. doi:10.1073/pnas.9.3.93.

2) Artin E. 1947. Theory of braids, Ann. of Math. 48.

3) Bailles A, Collinet C, Philippe J-M,Lenne P-F. Munro E, Lecuit T. 2019. Genetic induction and mechanochemical propagation of a morphogenetic wave. Nature. 572: 467-473.

4) Barriga EH, Franze K, Charras G, Mayor R. 2018. Tissue stiffening coordinates morphogenesis by triggering collective cell migration in vivo. Nature, 554: 523-527.

5) Bartolomei H, Kumar M, Bisognin R, Marguerite A, Berroir J-M, et al. 2020. Fractional statistics in anyon collisions.Science, Vol. 368, Issue 6487, pp. 173-177. DOI: 10.1126/science.aaz5601

6) Bhattacharya S, Iglesias PA. 2019. Controlling excitable wave behaviors through the tuning of three parameters. Biological Cybernetics, 113:61-70.

7) Birman JS. 1974. Knots, links, and mapping class groups. Annals of Math Study, no. 82, Princeton University Press. 
8) Boke E, Ruer M, Wühr M, Coughlin M, Lemaitre R, et al. 2016. Amyloid-like Self-Assembly of a Cellular Compartment. Cell;166(3):637-650. doi: 10.1016/j.cell.2016.06.051.

9) Box K, Joyce, BW, Devenport D. 2019. Epithelial geometry regulates spindle orientation and progenitor fate during formation of the mammalian epidermis. eLife 2019;8:e47102 DOI: 10.7554/eLife.47102

10) Brangwynne CP, Eckmann CR, Courson DS, Rybarska A, Hoege C, et al. 2009. Germline P Granules Are Liquid Droplets That Localize by Controlled Dissolution/Condensation. Science. Vol. 324, Issue 5935, pp. 1729-1732. DOI: 10.1126/science.1172046.

11) Brennen GK, Pachos JK. 2007. Why should anyone care about computing with anyons? Proceedings of the Royal Society A: Mathematical, Physical and Engineering Sciences,Volume 464, Issue 2089. https://doi.org/10.1098/rspa.2007.0026.

12) Chan CJ, Costanzo M, Ruiz-Herrero T, Mönke G, Petrie RJ, et al. 2019. Hydraulic control of mammalian embryo size and cell fate. Nature. 571: 112-116.

13) Chen VS, Morrison JP, Southwell MF, Foley JF, Bolon B, Elmore SA. 2017. Histology Atlas of the Developing Prenatal and Postnatal Mouse Central Nervous System, with Emphasis on Prenatal Days E7.5 to E18.5. Toxicol Pathol. 45(6):705-744. doi: 10.1177/0192623317728134.

14) Chvykov P, Berrueta TA, Vardhan A, Savoie W, Samland A, et al. 2021. Low rattling: A predictive principle for self-organization in active collectives. Science: Vol. 371, Issue 6524, pp. 90-95. DOI: 10.1126/science.abc6182.

15) Cromwell PR. 2004. Knots and Links. Cambridge, UK: Cambridge University Press.

16) Dabaghian Y, Brandt VL, Frank LM. 2014 Reconceiving the hippocampal map as a topological template. eLife 10.7554/eLife.03476. doi: http://dx.doi.org/10.7554/eLife.03476.

17) Dai W, Guo X, Cao Y, Mondo JA, Campanale JP, et al. 2020. Tissue topography steers migrating Drosophila border cells. Science. Vol. 370, Issue 6519, pp. 987-990. DOI: 10.1126/science.aaz4741.

18) Frieden BR, Gatenby RA. 2019. Signal transmission through elements of the cytoskeleton form an optimized information network in eukaryotic cells. Scientific Reports volume 9, Article number: 6110.

19) Greig JA, Nguyen TA, Lee M, Posey AE, Pappu RV, Jedd G. 2020. Arginine-Enriched Mixed-Charge Domains Provide Cohesion for Nuclear Speckle Condensation. Molecular Cell. 77(6):1237-1250.E4. DOI:https://doi.org/10.1016/j.molcel.2020.01.025.

20) Hartmann R, Singh PK, Pearce P, Mok R, Song B, et al. 2019. Emergence of three-dimensional order and structure in growing biofilms. Nature Physics, 15:251-256.

21) Herrera-Rincon C, Pai VP, Moran KM, Lemire JM, Levin M. 2017. The brain is required for normal muscle and nerve patterning during early Xenopus development. Nature Communications, volume 8, 587.

22) Imam N, Finlay BL. 2020. Self-organization of cortical areas in the development and evolution of neocortex. PNAS 117 (46) 29212-29220. https://doi.org/10.1073/pnas.2011724117.

23) Iqbal D. Samiullah MH. 2013. Photo-Responsive Shape-Memory and Shape-Changing Liquid-Crystal Polymer Networks. Materials. 6(1), 116-142; https://doi.org/10.3390/ma6010116.

24) Jizba P, Arimitsu T. 2001. The world according to Renyi: thermodynamics of fractal systems. AIP Conference Proceedings, 597, 341-348.

25) Jizba P., Korbel J. 2015. On q-non-extensive statistics with non-Tsallisian entropy. arXiv:1501.07386.

26) Kalmbach AS, Waters J. 2012. Brain surface temperature under a craniotomy. J Neurophysiol. 108(11), 3138-3146.

27) Kebschull JM, Richman EB, Ringach N, Friedmann D, Albarran E, et al. 2020. Cerebellar nuclei evolved by repeatedly duplicating a conserved cell-type set. Science. Vol. 370, Issue 6523, eabd5059. DOI: 10.1126/science.abd5059.

28) Kießling TR, Stange R, Käs JA, Fritsch AW. 2013. Thermorheology of living cells - impact of temperature variations on cell mechanics. New Journal of Physics, New J. Phys. 15045026.

29) Ladouceur A-M, Parmar BS, Biedzinski S, Wall J, Graydon Tope S, et al. 2020. Clusters of bacterial RNA polymerase are biomolecular condensates that assemble through liquid-liquid phase separation. PNAS, 117 (31) 18540-18549. https://doi.org/10.1073/pnas.2005019117.

30) Linneweber GA, Andriatsilavo M, Dutta SB, Bengochea M, Hellbruegge L, et al. 2020. A neurodevelopmental origin of behavioral individuality in the Drosophila visual system. Science. Vol. 367, Issue 6482, pp. 1112-1119. DOI: 10.1126/science.aaw7182.

31) Long MA, Fee MS. 2008. Using temperature to analyse temporal dynamics in the songbird motor pathway. Nature 456(7219):189-194.

32) Lovecchio E, Allegrini P, Geneston E, West BJ, Grigolini P. 2012. From self-organized to extended criticality. Front. Physiol., 26 April 2012 | https://doi.org/10.3389/fphys.2012.00098.

33) McDonald NA,Fetter RD, Shen K. 2020. Assembly of synaptic active zones requires phase separation of scaffold molecules. Nature, 588: 454-458.

34) Milton GW, Mattei O. 2017. Field patterns: a new mathematical object. Proceedings of the Royal Society A. Volume 473, Issue 2198. https://doi.org/10.1098/rspa.2016.0819.

35) Montis R, Fusaro L, Falqui A, Hursthouse MB, Tumanov N, et al. 2021. Complex structures arising from the self-assembly of a simple organic salt. Nature volume 590, pages275-278(2021) 
36) Mundoor H, Park S, Senyuk B, Wensink HH, Smalyukh II. 2018. Hybrid molecular-colloidal liquid crystals. Science, Vol. 360, Issue 6390, pp. 768-771. DOI: 10.1126/science.aap9359.

37) Mundoor H, Wu J-S, Wensink HH; Smalyukh II. 2021. Thermally reconfigurable monoclinic nematic colloidal fluids. Nature, 590, 268-274.

38) Nieuwenhuys R, Voogd J, van Huijzen C. 2008. The Human Central Nervous System. Fourth Edition, Springer Springer Berlin Heidelberg.

39) Nguyen TA, Le S, Lee M, Yang D, Yan J, Jedd G. 2020. Fungal Wound Healing through Instantaneous Protoplasmic Gelation. Current Biology, 31(2): 271-282.e5. DOI:https://doi.org/10.1016/j.cub.2020.10.016.

40) Xie M, Kamenev D, Kaucka M, Eleni Kastriti M, Zhou B, et al. 2019. Schwann cell precursors contribute to skeletal formation during embryonic development in mice and zebrafish. PNAS. 116 (30) 15068-15073. https://doi.org/10.1073/pnas.1900038116.

41) Xu Y, Ma J, Zhan X, Yang L, Jia Y. 2019. Temperature effect on memristive ion channels. Cognitive Neurodynamics volume 13: 601-611.

42) Qian Q, Nakamura J, Fallahi S, Gardner GC, Manfra MJ. 2017. Possible nematic to smectic phase transition in a two-dimensional electron gas at half-filling. Nature Communications volume 8, Article number: 1536.

43) Quiroz FG, Fiore VF, Levorse J, Polak L, Wong E, et al. 2020. Liquid-liquid phase separation drives skin barrier formation. Science. Vol. 367, Issue 6483, eaax9554. DOI: 10.1126/science.aax9554.

44) Nieuwenhuys R, Voogd J, Van Huijzen C. 2007. The Human Central Nervous System: A Synopsis and Atlas. Steinkopff Darmstadt; 4th edition. ISBN-10 : 3540346848 .

45) Reimann MW, Horlemann A-L, Ramaswamy S, Muller EB, Markram H. 2017. Morphological Diversity Strongly Constrains Synaptic Connectivity and Plasticity. Cereb Cortex;27(9):4570-4585. doi: 10.1093/cercor/bhx150. DOI: 10.1093/cercor/bhx150.

46) Shyer AE, Rodrigues AR, Schroeder GG; Kassianidou E, Kumar S, Harland RM. 2017. Emergent cellular self-organization and mechanosensation initiate follicle pattern in the avian skin. Science. Vol. 357, Issue 6353, pp. 811-815. DOI: 10.1126/science.aai7868

47) Soldatov R, Kaucka M, Kastriti ME, Petersen J, Chontorotzea T, et at. 2019. Spatiotemporal structure of cell fate decisions in murine neural crest. Science. Vol. 364, Issue 6444, eaas9536. DOI: 10.1126/science.aas9536.

48) Sotoma S, Zhong C, Kah JCY, Yamashita4 H. Plakhotnik T, et al. 2021. In situ measurements of intracellular thermal conductivity using heater-thermometer hybrid diamond nanosensors. Science Advances. Vol. 7, no. 3, eabd7888, DOI: 10.1126/sciadv.abd7888.

49) Strom AR, Emelyanov AV, Mir M, Fyodorov DV, Darzacq X, Karpen GH. 2017. Phase separation drives heterochromatin domain formation. Nature, 547:241-245.

50) Tanimoto R, Hiraiwa T. Nakai Y, Shindo Y,1 Oka K. et at. 2016. Detection of Temperature Difference in Neuronal Cells. Sci Rep.; 6: 22071. doi: 10.1038/srep22071.

51) Telley L, Agirman G, Prados J, Amberg N, Fièvre S, et al. 2019. Temporal patterning of apical progenitors and their daughter neurons in the developing neocortex. Science. Vol. 364, Issue 6440, eaav2522. DOI: 10.1126/science.aav2522.

52) Thompson DW. 1992. On Growth and Form. Dover Pubns. ISBN-10 : 0486671356.

53) Tozzi A. 2015. Information Processing in the CNS: A Supramolecular Chemistry? Cognitive Neurodynamics $9(5): 463-477$

54) Tozzi A. Peters JF. 2017. Critique of pure free energy principle: Comment on “Answering Schrödinger's question: A free-energy formulation" by Maxwell James Désormeau Ramstead et al. Physics of Life Reviews.DOI: 10.1016/j.plrev.2017.10.003.

55) Tozzi A, Peters JF, Ori O. 2017. Fullerenic-topological tools for honeycomb nanomechanics. Towards a fullerenic approach to brain functions. Fullerenes, Nanotubes and Carbon nanostructures.25 (4): 282-288. http://dx.doi.org/10.1080/1536383X.2017.1283618.

56) Veenvliet JV, Bolondi A, Kretzmer H, Haut L, Scholze-Wittler M, et al. 2020. Mouse embryonic stem cells self-organize into trunk-like structures with neural tube and somites. Science, Vol. 370, Issue 6522, eaba4937. DOI: $10.1126 /$ science.aba4937.

57) Wang H, Wang B, Normoyle KP, Jackson K, Spitler K, et al. 2014. Brain temperature and its fundamental properties: a review for clinical neuroscientists. Front. Neurosci., 8;8:307 2014 | doi: 10.3389/fnins.2014.00307.

58) Wu FY. 1992. Knot theory and statistical mechanics. Rev Mod Phys 64:1099-1131. 